\title{
Dynamics of Conflicts on the Twitter Social Network: a case study on the use of chloroquine in Brazil
}

\author{
Fabricio Olivetti de Franca \\ Federal University of ABC \\ folivetti@ufabc.edu.br \\ Carlos da Silva dos Santos \\ Claudio Luis C. Penteado \\ Denise Hideko Goya \\ Federal University of $\mathrm{ABC}$ Federal University of $\mathrm{ABC}$ \\ Federal University of ABC \\ carlos.ssantos@ufabc.edu.br claudio.penteado@ufabc.edu.br denise.goya@ufabc.edu.br \\ Lucas Mazim de Sousa \\ Daniel Vitor B. di Genova \\ Diogo Fornaziero S. Ramos \\ Carlos Alberto Kamienski \\ Federal University of ABC \\ Federal University of ABC \\ Federal University of ABC \\ Federal University of ABC \\ lucas.mazim@ufabc.edu.br daniel.genova@aluno.ufabc.edu.br diogo.ramos@ufabc.edu.br carlos.kamienski@ufabc.edu.br
}

\begin{abstract}
Concerns about the advances of the COVID-19 epidemic have sparked many debates around the world. One such discussion revolved around the use of the drug called chloroquine, initially thought to be effective in reducing the mortality rate of the infection. Particularly in Brazil, even after new studies pointed to the drug's ineffectiveness, the federal government kept the recommendation of this drug as an official treatment. The publication of an official authorization of the use of chloroquine on Twitter sparked an intense debate on social media with arguments against and in favor. This paper studies the dynamics of interactions among different user groups around this discussion, relying on network science and topic modeling analyses. Our results highlight two distinct behaviors in Twitter interaction networks, where retweets serve as positive reinforcements within information bubbles and replies act as a space of direct debate. Also, discussions are seeded by public figures, but regular users carry on the debate per se. The topic modeling analyses revealed three observable user groups in this debate: strong supporters of the Brazilian government, progressive opposition to this government, and moderate users that oppose to this specific topic but do not reject the government agenda as a whole.
\end{abstract}

\section{Introduction}

The COVID-19 pandemic sparked many debates about vaccine development, possible treatments, and non-pharmaceutical interventions such as social distancing. Discussions soon shifted to a political sphere caused by federal and state governments' conflicting actions such as sanitary measures, the shutdown of economic activities, and authorization for experimental treatments. One endeavor that mobilized researchers early on was the investigation of existent drugs. One such investigation was the treatment with Chloroquine (CQ) and hydroxychloroquine (HCQ). These drugs are currently used for the treatment of malaria and lupus and were candidates for such repurposing. By March 2020, early studies had revealed that CQ and HCQ could inhibit the infection of cells by SARS-CoV-2 in vitro [1], and a small observational study reported beneficial effects of HCQ for hospitalized COVID-19 patients [2].

In the United Kingdom, hydroxychloroquine was one of the drugs selected for the Randomised Evaluation of COVID-19 thERapY (RECOVERY) Trial that started enrolling patients by the end of March 2020. On March 20, 2020, the World Health Organization announced a sizeable global trial called Solidarity to test several known drugs for COVID-19 treatment, including chloroquine and hydroxychloroquine. On October 2, 2020, the website clinicaltrials.gov registered 508 studies related to hydroxychloroquine, out of 3,507 studies about SARS-CoV-2. In the absence of any proven treatment for COVID-19, the American FDA issued emergency authorization for chloroquine and hydroxychloroquine prescription in COVID-19 cases on March 28, 2020, revoked on June 15, 2020, as evidence mounted on the ineffectiveness of these drugs and their potentially dangerous side effects [3]. On June 5, 2020, preliminary results from the RECOVERY trial were announced, showing that hydroxychloroquine did not yield beneficial effects on hospital stay duration or other outcomes and concluded that HCQ treatment was indistinguishable from standard care [4]. The hydroxychloroquine arm of the RECOVERY trial was thus halted. On July 4, 2020, the World Health Organization decided to discontinue the chloroquine arm of the Solidarity trail [5], based on interim results that showed little to no effect in reducing mortality. The issue of CQ and HCQ prescription became politicized as soon as March 2020, as USA president Donald Trump 
recommended COVID-19 patients to take the drugs, despite the absence of concrete evidence and contrary to orientations by scientists [6].

In Brazil, president Bolsonaro followed up and embraced the defense of chloroquine prescription and refused restrictive measures such as quarantining and lockdown. Meanwhile, some state governors implemented social distance policies and ramped up the expansion of healthcare facilities, including ICU beds. In social media, many users engaged in the chloroquine debate and their political opinions, leading to heated discussions. The early interruption of a randomized trial of chloroquine [7] due to elevated mortality of patients led to social media controversy and death threats [8] to the scientists involved in the study, as digital media influencers and even one of the sons of the Brazilian president accused scientists of purposely administering unsafe dosages of the drug for alleged ideological motivations $[8,9]$.

This political debate depicts an intriguing aspect of our society wherein a group of people disbelieves scientific methods and their results and where people lacking specific knowledge can quickly become authorities. With this regard, Social Networks are used to spread facts and anti-facts to a broad population. One such network, Twitter, is an open space where we can observe these conflicts taking place. In Twitter, we have two different types of interactions: i) retweets, in which a message from a user is shared to all their followers, and, ii) replies, a direct form of interaction with the original poster. Retweets are mainly used to reinforce people's points of view, sharing what they want to believe $[10,11]$. On the other hand, replies can be used to express their agreement or counter-argue the original poster ideas [12].

The dynamics of Twitter, as much as other social networks, have been determinant to the results of the last political events in Brazil [13, 14] and points to a polarization of political debate on the Internet [15]. Political polarization on Twitter favors politicians with more extreme ideologies [16] and can help spread misinformation and distrust of public institutions, putting democracy at risk [17]. So far, many of these events were supported by personal political views from both sides, creating personal knowledge bubbles [18] and ideological conflicts that can lead to society's fragmentation [19]. In the particular debate on the use of chloroquine in Brazil, this ideological discussion involved supporters of the Brazilian president (favorable to the use of chloroquine), opposition groups, and defenders of the World Health Organization protocols to use a drug without scientific proof. This political conflict can create difficulties in developing proper healthy protocol as a response to a pandemic state, especially when this discussion comes from public authorities, like the president of the republic [20].

Studies on political polarization on social media have identified the importance of ideology as a factor of polarization and disinformation diffusion. However, there are still gaps for a better understanding of the phenomenon, such as the effect of polarization and disinformation on individuals' behavior and beliefs [17]. With the global COVID-19 pandemic, national governments adopted different measures to combat the new coronavirus. Research has emerged to study the role of ideological polarization in understanding these different reactions. Studies pointed out that ideological polarization in the USA has made it difficult to implement measures to combat COVID-19 [21, 22]. Another study proves the influence of ideology concerning the disregard of social distancing guidelines recommended by the World Health Organization [23]. On the other hand, studies showed a rare inter-party consensus (between political elites and the public) on important issues related to the COVID-19 pandemic, such as its severity and the need for social distance in Canada [24].

In a highly polarized social media ecosystem, monitoring and analyzing public debate and conflicting interactions on social network sites can assist public authorities in planning public health campaigns and identifying controversies around health procedures [22], with the identification of critical profiles in the debate and the main narratives mobilized by users.

In this paper, we study the dynamics of the social network interactions between different groups by using common network science metrics and topic modeling analysis. We seek to answer some research questions regarding reply and retweet networks extracted from the discussion about the Brazilian government's authorization to use the chloroquine for the COVID-19 treatment, despite the lack of conclusive studies of its effectiveness. Specifically, we want to answer:

- RQ 1: Which groups that take part in the debate around the authorization of the use of the chloroquine on Twitter? Are they representative of the partisanship currently established in Brazil?

- RQ 2: Who are the central users to these interactions (i.e., public figures or common people)?

- RQ 3: What are the discursive characteristics employed by each group in this conflict? 
This case study contributes to identifying the actors involved in online disputes and their discourse patterns in the construction of narratives between ideologically polarized groups on the subject of COVID-19, and forms of its treatment in social network sites.

The remainder of this paper is structured as follows. In Section 2 we explain our definition of conflicts on Social Networks and the influence of these events on society. Section 3 explains the methodology employed in this paper in order to answer the posed research questions, in Section 4 we report the results while providing some deeper analysis of our findings. Finally, in Section 5 we give final remarks together with some future research.

\section{Conflicts on Social Network}

The concept of social conflict is central to sociological theories, with different approaches that aim to explain the logic and dynamics of societies [25]. Despite being a typical human relationship, the conflict has a political dimension that affects society's dynamics and organization. This dimension is expressed in the dispute between the parties and groups. Beyond the political dimension, the conflicts on social networking sites have another two dimensions: communicational and sociotechnical. The former is related to the dispute around the meaning of an event or situation. In contrast, the latter refers to using online social network platforms as a conflict mediation device. Thus, the conflict on social internet networks reflects a discursive dispute through the interaction channels that operate within specific configurations. Although Twitter is not the most popular online social network in Brazil, due to its technical and usage characteristics, it is the main space for political conflict between different actors and a critical space for the study of public debate, where the main political information-producing agents publish their content [26]. On this platform, the main forms of interaction between profiles are retweet, reply, and likes. Through these functions, users can express their opinions and political positioning, agreeing or disagreeing with the content, promoting conflict relations.

Online Social Networks play a vital role in political dynamics in Brazil. During the impeachment process of former president Dilma Rousseff, different opinions emerged between 2015 and 2016, creating a bipartisan network of people denouncing the process as a coup and supporting the decisions [27]. The polarization on Twitter has intensified overtime during the 2018 Brazilian Presidential Election. In this election, social media was used on a large scale, particularly by
Bolsonaro and his supporters. The political debate on Twitter showed that the right-wing (Bolsonaros supporters) were more hyperpartisan outlets and shared more disinformation on Twitter than the left-wing group [28].

The strategy used by these groups to promote their point of view is twofold: tweet using a pre-specified hashtag to promote their topic of interest and retweet the messages published by authorities in their network. Authorities are users, public figures or not, whose opinions are considered trusted by a group. The promotion of their point of view influences people who do not have a formed opinion so far [29]. On some occasions, the groups may share posts from press vehicle accounts, adding a comment that either corroborates their opinion or criticizes the post. On some occasions, the original posts of news media or authorities are a seed of dispute between groups, in which they generate a thread of replies beating, demystifying the opposing arguments, or corroborating replies from people of the same group. While rarely changing the opinion of someone with a well-defined belief, the effect of these interactions can influence those who do not have a formed opinion. Our case study of the use of chloroquine may introduce peer pressure on public policy on the misuse of unproven treatments.

\section{Methodology}

For this study, we analyzed the interactions between different groups on Twitter after the Ministry of Health's announcement, authorizing the use of chloroquine treatment, disregarding the counter-evidence of different scientific publications. A total of 314,457 tweets were collected using the keyword cloroquina (chloroquine in Portuguese) within the period of 05/20/2020 at 5:48 pm to $05 / 21 / 2020$ at 8:23 pm (Brasilia local time - UTC -3).

From the raw dataset, we filtered those tweets that hold to at least one of these conditions: i) nonzero retweet count, ii) nonzero reply count, iii) nonempty in_reply_to field, iv) nonempty retweeted_from field. Afterwards we have selected the username and text fields from these tweets. The text field was normalized to lowercase and with any non-alphanumeric symbol removed. Afterward, any word with less than four characters was removed from the text. We then extract the binary relationship $R \subseteq U \times T$ of the set of users $U$ with the set of terms $T$ associating each user with the terms they used. This relationship will be used to find groups of users with similar discourse using a biclustering algorithm called HBLCoClust [30]. This algorithm extracts tuples $\left(U^{\prime}, T^{\prime}\right)$ with $U^{\prime} \subseteq U$ and $T^{\prime} \subseteq T$ such as $\forall(u, t) \in U^{\prime} \times T^{\prime},(u, t) \in R$. Each of 
these tuples can be interpreted as the users in $U^{\prime}$ belong to the same group when considering the set $T^{\prime}$ of terms.

In short, this algorithm starts by sampling different seed clusters using the Locality Sensitive Hashing technique [31]. Afterward, it applies the InClose algorithm [32] on each seed until it finds the maximal subsets $\left(U^{\prime}, T^{\prime}\right)$ containing this seed. This algorithm has two characteristics. First, it does not guarantee that a given user or term will be assigned to one of the clusters, which is an essential feature since some users utilized a very distinct set of terms that could be interpreted as noise in this stage. Second, it can find overlapping clusters, so any user or term can belong to more than one cluster. Each cluster was manually classified as in favor of the drug treatment, against it, or neutral by observing the subset of terms. Those users that belonged to clusters of the same label were selected as seed users.

From the processed data set, we extracted the Reply Network (RPNet), a directed network with an edge from $A$ to $B$ representing that user $A$ replied to user $B$, and the Retweet Network (RTNet), with an edge from $A$ to $B$ representing that user $A$ retweeted a message from user $B$. Finally, we eliminated every connected component of the networks that did not contain any of the seed users. The nodes of these networks were manually labeled as their polarity: Bolsonarist (in agreement with the Bolsonaro's government), Progressist (in total disagreement with the government), Moderate (in disagreement with the government w.r.t. this topic), NA (banned or deleted accounts). The classification process took into account the users' history of tweets, not limited to the tweets about chloroquine.

The assortativity of user polarity identifies the intensity of conflict in each network, where assortativity is the average pairwise correlation of the connections between different labels. A positive value means that a network contains interactions between users of the same polarity, while negative values indicate a larger number of interactions among users with different polarities. A value close to 0 means that there is no observable interaction (i.e., they happen at random). We also ranked the top ten users, according to the in-degree, out-degree, and PageRank centrality metrics. The in-degree reveals users who attract many replies or retweets, while the out-degree reveals users who are very active in replying or retweeting. PageRank [33] reveals users who are replied/retweeted by users that also receive a large number of replies or retweets. Apart from this user-centric analysis, we will also apply the Latent Dirichlet Allocation [34] algorithm to extract the main topics from the data set and find the similarities and dissimilarities of discourse between groups.
Table 1. Summary of the network properties.

\begin{tabular}{ccc}
\hline & RTNet & RPNet \\
\hline Nodes & 196 & 1632 \\
Edges & 204 & 1682 \\
Bolsonarists & $44.39 \%$ & $53.92 \%$ \\
Moderates & $8.16 \%$ & $11.76 \%$ \\
Progressist & $26.02 \%$ & $15.2 \%$ \\
NA & $21.43 \%$ & $11.34 \%$ \\
\hline
\end{tabular}

\section{Results and Analysis}

We applied the biclustering algorithm constraining the clusters' size to a minimum of 5 users and 5 terms per cluster. With this configuration, we found 17 clusters covering a total of $1.32 \%$ of the 17,680 users.

Table 1 shows the main properties of the generated networks. As we can see from this table, the number of edges and nodes is very close for both networks, a side effect of the formation of the network as a set of threads. Overall, most users in both networks are labeled as Bolsonarists, while Progressists and Unavailable accounts are more prominent in the retweet network. While Bolsonarists account for approximately half of the interactions in both networks, the Progressists seem to be more engaged in indirect attacks through the retweets. We have noticed from a manual inspection in this and other similar datasets that these users engage in some trending topics war in which one side generates some trend by posting many tweets with a given hashtag, and the other side hijacks this hashtag by posting mockup tweets.

The reply network has an assortativeness of -0.18 , while the retweet network has a value of 0.31 . These results corroborate with the intuition that the discussions take place in the reply network and, even though there is still some reinforcement of ideas in this network, the predominance is of direct attack.

Table 2 describes the main actors of this network based on their degree centrality, illustrated later in this same section. Notice that, even though SF_Moro is labeled moderate, he was formerly a strong supporter of the government. Another interesting finding is the presence of the EdPazuello account, an anonymous impersonator for the minister of Health, Eduardo Pazuello. That account achieves high visibility among rightwing groups for a short period, before being removed as the ministry of health denied any relation to it.

Figures 1 and 2 show the RTNet and RPNet, respectively. We can see two main communities on RTNet, one composed of users labeled as Bolsonarists 
Table 2. Main actors of RPNet and RTNet. Those against the use of chloroquine are marked in bold.

\begin{tabular}{|c|c|c|}
\hline Username & Polarity & Description \\
\hline jairbolsonaro & Bolsonarist & Brazilian President. \\
\hline XadrezVerbal & Progressist & $\begin{array}{l}\text { Humorous profile } \\
\text { that addresses issues } \\
\text { related to history and } \\
\text { current events. }\end{array}$ \\
\hline EdPazuello & Bolsonarist & $\begin{array}{l}\text { Impersonator of } \\
\text { interim minister } \\
\text { of health Eduardo } \\
\text { Pazuello (who is } \\
\text { also a general of the } \\
\text { Brazilian Army) }\end{array}$ \\
\hline bolsonarosp & Bolsonarist & $\begin{array}{l}\text { Congressman; Son of } \\
\text { the President. }\end{array}$ \\
\hline samiabomfim & Progressist & $\begin{array}{l}\text { Congresswoman of } \\
\text { the opposition. }\end{array}$ \\
\hline joseserra_ & Moderate & $\begin{array}{l}\text { Senator and supporter } \\
\text { of liberal politics. }\end{array}$ \\
\hline LucianoHuck & Moderate & $\begin{array}{l}\text { Popular TV host and } \\
\text { supporter of liberal } \\
\text { politics. }\end{array}$ \\
\hline SF $\perp$ Moro & Moderate & $\begin{array}{l}\text { Former judge and } \\
\text { Minister of Justice } \\
\text { of the Bolsonaro } \\
\text { government; famous } \\
\text { for arresting former } \\
\text { president Lula; left } \\
\text { the government as } \\
\text { he raised serious } \\
\text { accusations against } \\
\text { Bolsonaro. }\end{array}$ \\
\hline
\end{tabular}

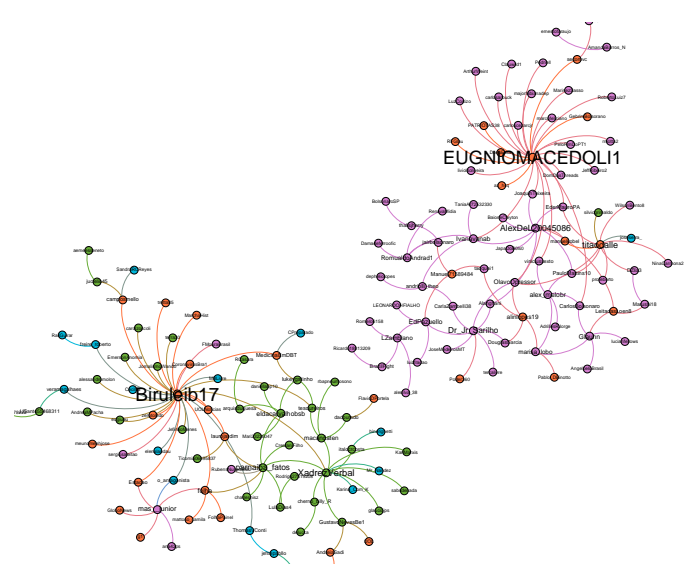

Figure 1. Retweet network (RTNet). Bolsonarists are colored purple, Progressists are green, Moderates are blue and unavailable accounts are red.

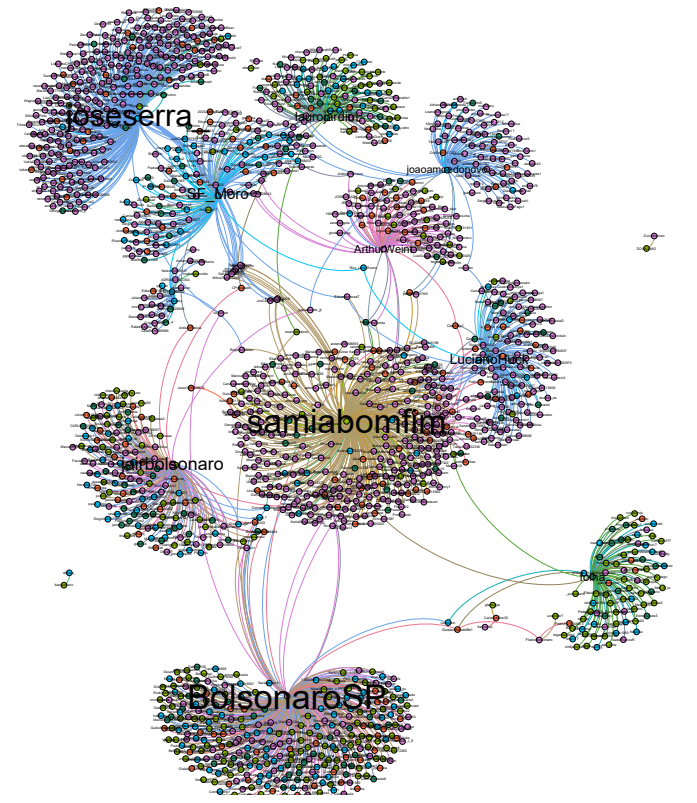

Figure 2. Reply network (RPNet). Bolsonarists are colored purple, Progressists are green, Moderates are blue and unavailable accounts are red.

and another with a mix of Progressists and Moderates. This happened because the Progressists and Moderates share the same perspective on this topic, although using different discourses, as we will see in the next sections.

The RPNet, on the other hand, depicts an interesting pattern with many communities. Although having nodes of different labels, each community reveals users who predominantly oppose the original poster. For example, the nodes belonging to communities centered around jairbolsonaro and bolsonaroSP, both Bolsonarists, are predominantly progressists and moderates, while the community centered around samiabomfim (progressist), LucianoHuck (moderate), and joseserra_ (moderate) are predominantly labeled as Bolsonarists. One exception is $S F$ Moro (moderate), who has a heterogeneous set of connections. Also, his tweets received criticisms from Bolsonarists accusing him of turning his back on the government and from progressists accusing him of being quiet about the government for this whole time.

As some illustrative examples, the replies to bolsonaroSP tweet are direct name callings and mentions of open cases accusing the congressman of corruption. Meanwhile, the answers to the congresswoman samiabomfim also are mostly name callings and some mockeries. Curiously, the replies to posts from moderate accounts (LucianoHuck, joseserra_) are also more moderates. They are either challenging them not to take chloroquine if they ever get sick or refuting their opinion with fake information 
on the use of chloroquine around the world.

Tables 3 and 4 depict the most retweeted and replied tweets, respectively. The first retweet from Table 3 is a satirical aggregation of memes mocking some of the recent Bolsonarists posts about a made-up story of some guy that died because of a burst tire, but his cause of death was marked as COVID-19. It also mentions a hoax of a miracle combination of drugs that healed someone in less than five days. Again, there is the cancellation of Dell for stopping advertising on some sites accused of spreading fake news. The second most retweeted tweet was made by one account impersonating the (then) interim Ministry of Health, general Eduardo Pazuello, announcing the authorization of the use of chloroquine for the treatment of COVID-19 patients.

Regarding the tweets with most replies, the first one was made by the son of the president complimenting the Ministry of Health decision, stating that millions of lives would be saved with this decision. The second one, made by an opposing party congresswoman, criticizes the act.

Observing more samples from the most retweeted and most replied, we can see that the retweets are either announcements or mockeries attacking the opposing party. The topics of replies, on the other hand, revolves around more critical tweets. This can also be observed with the fact that the most replied users (see Figure 3) are public figures, while most RT users (see Figure 4) are mostly composed of ordinary people supporting either side.

Table 3. Sample tweets from RTNet.

\begin{tabular}{|c|c|}
\hline User & Tweet (adapted) \\
\hline XadrezVerbal & $\begin{array}{l}\text { The doormans cousin of my } \\
\text { building was changing his Dells } \\
\text { tires and it burst in my daughters } \\
\text { face that got infected by COVID, } \\
\text { but they gave her AZT+HCQ+Zinc } \\
\text { and after } 4 \text { days the tire was brand } \\
\text { new \#DoNotBuyDell }\end{array}$ \\
\hline EdPazuello & $\begin{array}{l}\text { After two Ministries of Health } \\
\text { refused to sign this document, } \\
\text { general Eduardo Pazuello signed } \\
\text { the authorization of the use of } \\
\text { chloroquine. }\end{array}$ \\
\hline
\end{tabular}

\subsection{Centrality Measures}

In Figures 3 and 4, we show the relative rankings of the top-10 users for the In-Degree and Pagerank centrality for the RPNet and RTNet, respectively. In
Table 4. Sample tweets from RPNet.

\begin{tabular}{|c|c|}
\hline User & Tweet (adapted) \\
\hline bolsonaroSP & $\begin{array}{l}\text { Today@JairBolsonaro took a brave } \\
\text { decision that will save millions } \\
\text { of lives: he authorized the use } \\
\text { of chloroquine for all COVID-19 } \\
\text { cases, even the brand ones. }\end{array}$ \\
\hline samiabomfim & $\begin{array}{l}\text { Bolsonaro finally forced the } \\
\text { Ministry of Health to authorize } \\
\text { the use of chloroquine. The } \\
\text { patients will have to sign a term of } \\
\text { agreement stating that there is no } \\
\text { proof of the medicines efficacy and } \\
\text { that it can even kill. Its an attempt } \\
\text { against the lives of Brazilians. }\end{array}$ \\
\hline
\end{tabular}

these figures, we labeled the users as a politician $(\mathrm{P})$, journalist $(\mathrm{J})$, media $(\mathrm{M})$, twitter $(\mathrm{T})$, celebrities (A), common user (U), other profiles $(\mathrm{O})$, and unavailable accounts $(\mathrm{N})$. The twitter label represents users with more than 1,000 followers, common user are those users who do not attend any of these criteria, and other profiles are those that were not possible to classify since they are a mix of one or more labels.

Apart from what we have reported so far, we can see from these tables that the in-degree is often represented by public figures in RPNet, and a variety of different profiles, in RTNet. The out-degree is composed of common users in both networks. An interesting result from Figure 3 can be observed from the comparison of in-degree and PageRank. The users joseserra_ and folha, both moderates in this context, were promoted to the highest rank in PageRank. This happened for two reasons: i) they were replied by users that also received many replies and, ii) their thread is perceived as a safe ground for both Bolsonarists and Progressists to discuss, creating a pattern of a given user A replying to B and generating lots of replies, inflating the authority measure of user B.

The first reason indicates a particular hierarchy of interactions in which popular users express a preference of replying to other popular users, while common users tend to reply directly to the original poster. These direct replies to politics and public figures are often carried on by militants [35] and robots [36, 37, 38, 13] that are mobilized to make their counter-arguments more visible. We will leave this analysis for future research since the collected data does not contain any side information necessary to identify robots [13].

In Fig. 4, we observe a different dynamics in the comparison of In-Degree and PageRank, where 


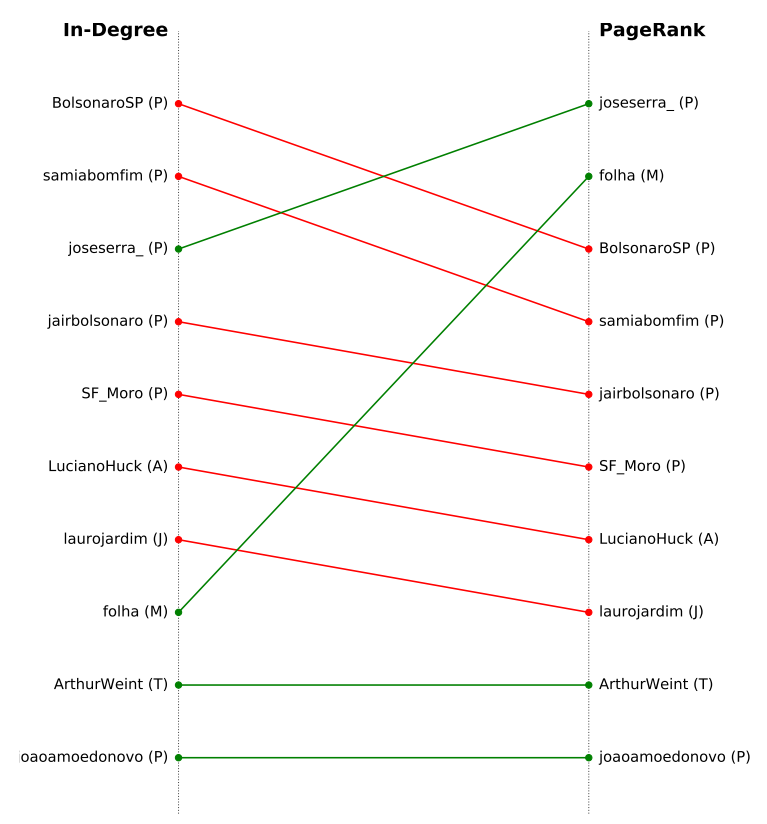

Figure 3. Rank of the top-10 users from RPNet with respect to in-Degree and PageRank.

we observe a difference in users that appear in the In-Degree and PageRank columns. The users in the set difference between PageRank column and In-Degree are accounts followed by different groups such as right wing extremists, medicine news venues, and journalists.

Finally, in Fig. 5, we can see the proportion of different types of users in the top-10 rank for the Out-Degree in both networks, the $y$-axis represents the number of each type of users that appeared in the top- 10 Out-degree list. This figure shows that common users account for most of the top-10 in both networks. They diverge on the second most frequent type of accounts where Twitters are the most common in RPNet and Not Available in RTNet. We theorize that these Not Available accounts are robots banned by Twitter, which will be investigated in future research.

\subsection{Topic Modeling}

To investigate the discourse patterns among different groups, we generated a topic model for the tweets dataset using the Latent Dirichlet Allocation (LDA) technique. The model was trained on the full dataset, resulting in 9 topics. We assigned a short explanation for all topics by inspecting the key terms and the top tweets of each topic (i.e., those with the highest probability for a specific topic). Table 5 shows the interpretation of the topics and the number of tweets attributed to each one. Table 6 shows the distribution of tweets among groups discriminated by topic: given a group $g$ and a topic $t$, we

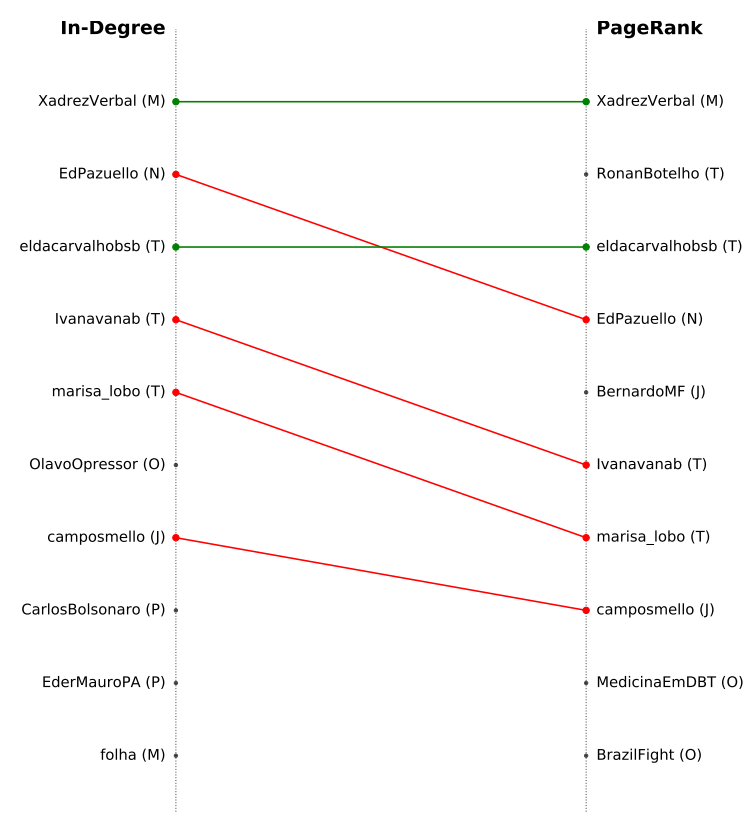

Figure 4. Rank of the top-10 users from RTNet with respect to in-Degree and PageRank.

calculated the probability $P(g \mid t)$ of finding a tweet by a member of the group $g$ among tweets labeled as topic $t$.

Overall, the supporters of the Brazilian president account for about half of the tweets. The results show that Bolsonarists tend to dominate topics (3 and 5) that reinforce the government's agenda. For instance, $73 \%$ of the activity around topic 3 was generated by Bolsonarist (B) accounts, echoing a video of the president mocking the opposition. Moderates generated $13 \%$ of the activity around the topic 3 , while Progressives (P) were responsible for $14 \%$. The participation of Progressists is increased when a topic is explicitly antagonistic towards the government $(6,8$, 9 ). Moderates (M) participated more in topics related to the drug's side effects and procedural aspects of its authorization $(1,7)$. These observations reinforce that Progressives and Moderates shared some viewpoints in this matter, but on average, Moderates still participated less in outright attacks against the government. While the LDA Topic Model shows differences of discourse between groups, it is not sufficient to separate them, as all groups engage in discussion on every topic.

By inspecting the top tweets related to each topic, we identified some recurring themes within groups. Distrust of pharmaceutical companies is a common point between Progressist and Bolsonarist discourse. However, while Progressists point to supposed links between the government and owners of chemical laboratories that could benefit from a surge in chloroquine production, Bolsonarists claim 


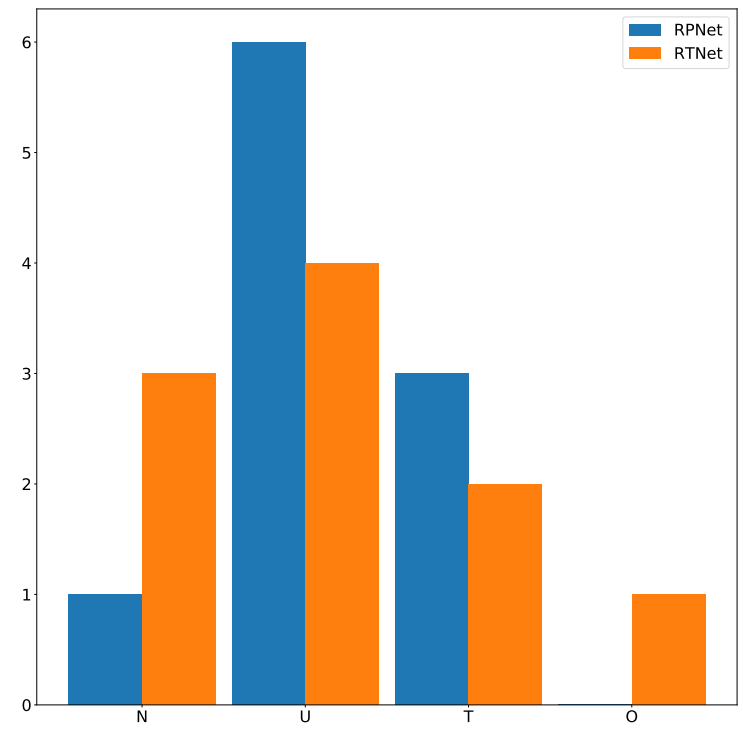

Figure 5. Ratio of different types of users in out-Degree.

that big pharmaceutical companies oppose chloroquine treatments due to the lack of patents over this drug from which companies could profit. Both Progressists and Moderates emphasize the absence of scientific evidence for chloroquine efficacy for COVID-19 and the potential for side effects, such as arrhythmia. Bolsonarist tweets display a variety of counterarguments. Bolsonarists often claim that CLQ and HCQ are safe drugs, used regularly to treat lupus and malaria. When confronted with the lack of evidence for the efficacy of the drugs, Bolsonarists respond that failed trials did not treat patients early enough and that CLQ and HCQ should be combined with other drugs, such as Zync and Azithromycin, for better effect. Sometimes, those claims reference preprint articles that would supposedly confirm the advantages of chloroquine treatments. This highlights one characteristic of social media discussions of COVID-19. Interest in the disease caused an explosion in the number of studies circulating on social media, peer-reviewed, and uploaded to preprint servers. In chloroquine treatments, many of these studies suffer from low statistical power and data selection effects. This allows one to cherry-pick studies to build arguments online while retaining the appearance of scientific rigor.

\section{Conclusion}

In this paper, we studied the differences between two interaction networks formed on Twitter during the debate revolving around the use of chloroquine in Brazil,
Table 5. Interpretation of topics.

\begin{tabular}{|c|c|c|}
\hline Topic & \# tweets & Interpretation \\
\hline 1 & 507 & $\begin{array}{l}\text { Role of Ministry of Health in } \\
\text { defining drug use protocol; possible } \\
\text { drug side effects; more technical } \\
\text { debate. }\end{array}$ \\
\hline 2 & 422 & Discussion of drug side effects. \\
\hline 3 & 270 & $\begin{array}{l}\text { Reaction to president Bolsonaro } \\
\text { speech. }\end{array}$ \\
\hline 4 & 264 & $\begin{array}{l}\text { Politics of CLQ use authorization, } \\
\text { involving the Supreme Court. }\end{array}$ \\
\hline 5 & 164 & $\begin{array}{l}\text { Accusations against governors that } \\
\text { supposedly oppose CLQ use. }\end{array}$ \\
\hline 6 & 136 & $\begin{array}{l}\text { Accusations of corporate interests } \\
\text { behind drug recommendations. }\end{array}$ \\
\hline 7 & 91 & $\begin{array}{l}\text { Short statements in support/against } \\
\text { CLQ use. }\end{array}$ \\
\hline 8 & 89 & $\begin{array}{l}\text { Mockery of and offensive comments } \\
\text { towards the other party. }\end{array}$ \\
\hline 9 & 83 & $\begin{array}{l}\text { Media story of president } \\
\text { possible interference on drug } \\
\text { recommendations, } \\
\text { against the press. }\end{array}$ \\
\hline
\end{tabular}

recently authorized by the federal government. For this study, we collected roughly 26 hours of tweets using the keyword cloroquina. Afterward, we processed those tweets to identify the key reply and retweet networks debating this topic. First, we applied a clustering algorithm to the tweets' texts to identify seed users selected based on their speech (i.e., always with the same point of view). Then, we extracted all the interaction networks containing such users. Finally, we applied some network science metrics to these networks and topic modeling analysis to their tweets.

From the network science perspective, we verified through the polarization assortativeness of these networks that users tend to interact in the retweet

Table 6. Distribution of topics.

\begin{tabular}{cccc}
\hline Topic $(t)$ & $p(B \mid t)$ & $p(M \mid t)$ & $p(P \mid t)$ \\
\hline 1 & 0.43 & 0.35 & 0.22 \\
2 & 0.64 & 0.18 & 0.18 \\
3 & 0.73 & 0.13 & 0.14 \\
4 & 0.57 & 0.21 & 0.22 \\
5 & 0.68 & 0.17 & 0.16 \\
6 & 0.34 & 0.32 & 0.35 \\
7 & 0.32 & 0.44 & 0.24 \\
8 & 0.29 & 0.29 & 0.42 \\
9 & 0.41 & 0.28 & 0.30 \\
\hline
\end{tabular}


network to reinforce their beliefs and tend to dispute in the reply network. Through PageRank, we also identified a higher impact on the discussions revolving around news media and moderate politicians. We theorize that they can be perceived as a middle ground where both groups use to publish their views. Another interesting observation is that the public figures seem to start the debate but not participate in the following discussions. Most discussion is carried on by common users, suggesting that the country still lives a high degree of polarization as studied in [27].

From the point of view of Political Science, our findings corroborate with the existence of ideological political polarization in digital networks [15] and its influence on the debate of issues that should be debated without a political point of view. This political and ideological conflict reflects the difficulty in implementing measures to combat CODIV-19 [21, 22]. In Brazil, supporters of President Bolsonaro (chloroquine advocates) will celebrate the approval of the drug by the Ministry of Health. On the other hand, critics groups will gather profiles that oppose the government (progressists) and those that only partially support it (moderates), signaling the antagonistic character of politics, especially in the discursive field [39]. A topic modeling analysis showed that Progressists and Moderates were united in highlighting the lack of evidence for drug therapies and their potential dangers. Bolsonarists celebrated the decision to allow chloroquine prescription and insisted on its safety. We also observed that Bolsonarists and Progressists used offensive language more often than Moderates.

As a general contribution to the network science community, this same pipeline can be applied to similar datasets with conflicting opinions. Notably, the clustering step helped us to focus on the region of the data containing the debate. This methodology still has some limitations as it depends on a consistent pattern of speech from the users in which each group uses a set of terms distinct from other groups. Also, the results obtained here are limited to this single case study, as we have yet to apply this same methodology into different contexts to verify the consistency of our findings.

As future work, we intend to devise a methodology for partially automating the user labeling process. We theorize that the retweet network's interactions can propagate the label of the original posting user to their direct interactions. Another step is applying this methodology to other debates, not necessarily political, to verify if the same methodology renders similar results in different contexts. Finally, we will study the temporal dynamics of retweet and reply networks when there is polarization around a certain topic.

\section{Acknowledgment}

This project is funded by Fundação de Amparo à Pesquisa do Estado de São Paulo (FAPESP), grant number 2018/23022-3.

\section{References}

[1] J. Liu, R. Cao, M. Xu, X. Wang, H. Zhang, H. Hu, Y. Li, Z. Hu, W. Zhong, and M. Wang, "Hydroxychloroquine, a less toxic derivative of chloroquine, is effective in inhibiting SARS-CoV-2 infection in vitro," Cell discovery, vol. 6, no. 1, pp. 1-4, 2020.

[2] P. Gautret, J.-C. Lagier, P. Parola, L. Meddeb, M. Mailhe, B. Doudier, J. Courjon, V. Giordanengo, V. E. Vieira, H. T. Dupont, et al., "Hydroxychloroquine and azithromycin as a treatment of COVID-19: results of an open-label non-randomized clinical trial," International journal of antimicrobial agents, p. 105949, 2020.

[3] US Food and Drug Administration and others, "Coronavirus (COVID-19) update: FDA revokes emergency use authorization for chloroquine and hydroxychloroquine," 2020.

[4] P. Horby, M. Mafham, L. Linsell, J. L. Bell, N. Staplin, J. R. Emberson, M. Wiselka, A. Ustianowski, E. Elmahi, B. Prudon, A. Whitehouse, T. Felton, J. Williams, J. Faccenda, J. Underwood, J. K. Baillie, L. Chappell, S. N. Faust, T. Jaki, K. Jeffery, W. S. Lim, A. Montgomery, K. Rowan, J. Tarning, J. A. Watson, N. J. White, E. Juszczak, R. Haynes, and M. J. Landray, "Effect of hydroxychloroquine in hospitalized patients with COVID-19: Preliminary results from a multi-centre, randomized, controlled trial.," medRxiv, 2020.

[5] "Solidarity clinical trial for COVID-19 treatments." https://www.who.int/emergencies/ diseases/novel-coronavirus-2019/ global-research-on-novel-coronavirus2019-ncov/solidarity-clinical-trialfor-covid-19-treatments. Accessed: 2020-07-10.

[6] D. G. K. Thomas, "Trumps embrace of unproven drugs to treat coronavirus defies science." https: //www.nytimes.com/2020/03/20/health/ coronavirus-chloroquine-trump.html. Accessed: 2020-07-10.

[7] M. G. S. Borba, F. F. A. Val, V. S. Sampaio, M. A. A. Alexandre, G. C. Melo, M. Brito, M. P. G. Mouro, J. D. Brito-Sousa, D. Baa-da Silva, M. V. F. Guerra, L. A. Hajjar, R. C. Pinto, A. A. S. Balieiro, A. G. F. Pacheco, J. Santos, James Dean Oliveira, F. G. Naveca, M. S. Xavier, A. M. Siqueira, A. Schwarzbold, J. Croda, M. L. Nogueira, G. A. S. Romero, Q. Bassat, C. J. Fontes, B. C. Albuquerque, C.-T. Daniel-Ribeiro, W. M. Monteiro, M. V. G. Lacerda, and for the CloroCovid-19 Team, "Effect of High vs Low Doses of Chloroquine Diphosphate as Adjunctive Therapy for Patients Hospitalized With Severe Acute Respiratory Syndrome Coronavirus 2 (SARS-CoV-2) Infection: A Randomized Clinical Trial," JAMA Network Open, vol. 3, pp. e208857-e208857, 042020.

[8] E. Ektorp, "Death threats after a trial on chloroquine for covid-19," The Lancet Infectious Diseases, vol. 20, p. 661, Jun 2020. 
[9] L. Wessel, "Its a nightmare. how Brazilian scientists became ensnared in chloroquine politics," June 2020 doi:10.1126/science.abd4620.

[10] M. D. Valle and R. Bravo, "Echo chambers in parliamentary twitter networks: The Catalan case," Int J Communication, no. 1, 2018.

[11] D. Boyd, S. Golder, and G. Lotan, "Tweet, tweet, retweet: Conversational aspects of retweeting on twitter,' in 2010 43rd Hawaii International Conference on System Sciences, pp. 1-10, 2010.

[12] J. Schantl, R. Kaiser, C. Wagner, and M. Strohmaier, "The utility of social and topical factors in anticipating repliers in twitter conversations," in Proceedings of the 5th Annual ACM Web Science Conference, WebSci '13, (New York, NY, USA), p. 376385, Association for Computing Machinery, 2013.

[13] . T. de Oliveira, F. de Frana, D. Goya, and C. Penteado, "The influence of retweeting robots during Brazilian protests," in 2016 49th Hawaii International Conference on System Sciences (HICSS), pp. 2068-2076, 012016.

[14] F. de Frana, D. Goya, and C. Penteado, "Analysis of the twitter interactions during the impeachment of Brazilian president," in 2018 Hawaii International Conference on System Sciences (HICSS), 012018.

[15] L. A. Adamic and N. Glance, "The political blogosphere and the 2004 U.S. election: Divided they blog," in Proceedings of the 3rd International Workshop on Link Discovery, LinkKDD '05, (New York, NY, USA), p. 3643, Association for Computing Machinery, 2005.

[16] S. Hong and S. Kim, "Political polarization on twitter: Implications for the use of social media in digital governments," Government Information Quarterly, vol. 33, pp. 777-782, Oct. 2016.

[17] J. A. Tucker, A. Guess, P. Barbera, C. Vaccari, A. Siegel, S. Sanovich, D. Stukal, and B. Nyhan, "Social media, political polarization, and political disinformation: A review of the scientific literature," 2018. Available at SSRN: https://ssrn.com/abstract=3144139 or http://dx.doi.org/10.2139/ssrn.3144139.

[18] N. Gillani, A. Yuan, M. Saveski, S. Vosoughi, and D. Roy, "Me, my echo chamber, and I: Introspection on social media polarization," in Proceedings of the 2018 World Wide Web Conference, WWW'18, (Republic and Canton of Geneva, CHE), p. 823831, International World Wide Web Conferences Steering Committee, 2018.

[19] C. R. Sunstein, \# Republic: Divided democracy in the age of social media. Princeton University Press, 2018.

[20] T. L. Brunell and S. P. Maxwell, "How partisanship affected public reaction to potential treatments for COVID-19," World Medical \& Health Policy, vol. n/a, no. n/a, 2020.

[21] J. Green, J. Edgerton, D. Naftel, K. Shoub, and S. J. Cranmer, "Elusive consensus: Polarization in elite communication on the COVID-19 pandemic," Science Advances, vol. 6, no. 28, 2020.

[22] J. Jiang, E. Chen, S. Yan, K. Lerman, and E. Ferrara, "Political polarization drives online conversations about COVID-19 in the United States," Human Behavior and Emerging Technologies, vol. 2, no. 3, pp. 200-211, 2020.

[23] C. A. Harper and D. Rhodes, "Ideological responses to the breaking of COVID-19 social distancing recommendations," August $2020 . \quad$ Available: psyarxiv.com/dkqj6.
[24] E. Merkley, A. Bridgman, P. J. Loewen, T. Owen, D. Ruths, and O. Zhilin, "A rare moment of cross-partisan consensus: Elite and public response to the COVID-19 pandemic in Canada," Canadian Journal of Political Science, vol. 53, no. 2, p. 311318, 2020.

[25] P. Birnbaum, "Conflitos," in Tratado de Sociologia (R. Boudon, ed.), pp. 243-282, Rio de Janeiro: Jorge Zahar Editora, 1995.

[26] K. Weller, A. Bruns, J. Burgess, M. Mahrt, and C. Puschmann, Twitter and society. Peter Lang Publishing, 012014.

[27] C. de Souza Carvalho, F. de Frana, D. Goya, and C. Penteado, "Brazilians divided: Political protests as told by twitter," in Transactions on Large-Scale Data-and Knowledge-Centered Systems XXVII, vol. 9860, pp. 1-18, 092016.

[28] R. Recuero, F. B. Soares, and A. Gruzd, "Hyperpartisanship, disinformation and political conversations on twitter: The Brazilian presidential election of 2018," Proceedings of the International AAAI Conference on Web and Social Media, vol. 14, pp. 569-578, May 2020.

[29] E. Bakshy, I. Rosenn, C. Marlow, and L. Adamic, "The role of social networks in information diffusion," in WWW'12 - Proceedings of the 21st Annual Conference on World Wide Web, 012012.

[30] F. O. de Frana, "A hash-based co-clustering algorithm for categorical data," Expert Systems with Applications, vol. 64, pp. 24-35, 2016.

[31] P. Indyk and R. Motwani, "Approximate nearest neighbors: Towards removing the curse of dimensionality," in Proceedings of the Thirtieth Annual ACM Symposium on Theory of Computing, STOC '98, (New York, NY, USA), p. 604613, Association for Computing Machinery, 1998.

[32] S. Andrews, "In-close, a fast algorithm for computing formal concepts," in International Conference on Conceptual Structures (ICCS), pp. 1-15, January 2009.

[33] D. Sullivan, "What is Google PageRank? a guide for searchers \& webmasters," Search engine land, 2007.

[34] D. Blei, A. Ng, and M. Jordan, "Latent Dirichlet allocation," Journal of Machine Learning Research, vol. 3, pp. 993-1022, 012003.

[35] V. Crosset, S. Tanner, and A. Campana, "Researching far right groups on twitter: Methodological challenges 2.0," New media \& society, vol. 21, no. 4, pp. 939-961, 2019.

[36] V. do Nascimento Silva and R. H. A. Silva, "Are algorithms affecting the democracy in Brazil?," in International Symposium on Ethical Algorithms, 2019.

[37] J. Messias, L. Schmidt, R. Oliveira, and F. Benevenuto, "You followed my bot! transforming robots into influential users in twitter," First Monday, vol. 18, Jun. 2013.

[38] T. Lokot and N. Diakopoulos, "News bots: Automating news and information dissemination on twitter," Digital Journalism, vol. 4, no. 6, pp. 682-699, 2016.

[39] C. Mouffe et al., On the political. Psychology Press, 2005. 